\title{
Imaging methods in primary Sjögren's syndrome as potential tools of disease diagnostics and monitoring
}

\author{
Marta Świecka ${ }^{1}$, Maria Maślińska ${ }^{1}$, Łukasz Paluch ${ }^{2}$, Jakub Zakrzewski ${ }^{2}$, Brygida Kwiatkowska ${ }^{1}$ \\ ${ }^{1}$ Clinic of Early Arthritis, National Institute of Geriatrics, Rheumatology and Rehabilitation, Warsaw, Poland \\ 2Department of Radiology, Gruca Orthopedic and Trauma Teaching Hospital, Center of Postgraduate Medical Education, \\ Otwock, Poland
}

\begin{abstract}
Primary Sjögren's syndrome (pSS) is a systemic autoimmune disease that affects exocrine glands, especially salivary and lacrimal glands, leading to their progressive destruction. With highly differentiated clinical course the approach to the definition of pSS is constantly evolving and the pSS classification criteria have been modified over the past years. In the past sialography and scintigraphy were frequently used in pSS diagnostics. Recently no imaging method has been included in the pSS classification criteria. That raises the question about the benefits of using novel imaging techniques such as ultrasonography, elastography and magnetic resonance imaging to diagnose and monitor pSS patients. The purpose of this review is to evaluate the advantages and limitations of widely used imaging methods in PSS and their possible future development.
\end{abstract}

Key words: Sjögren’s syndrome, elastography, ultrasonography, imaging diagnostics.

\section{Introduction}

Primary Sjögren's syndrome (pSS) is one of the most common autoimmune inflammatory rheumatic diseases. With its chronic course the disease has a destructive effect upon salivary and lacrimal glands, causing xerostomia and keratoconjunctivitis sicca. Some pSS patients have systemic organ involvement, and in the course of pSS the risk of lymphoma development is increased [1]. That is why it is vital to diagnose and treat the disease without delay.

Over the years the attempt to classify the disease has changed. Between 1965 and 2012, 12 various classifications and/or diagnostic criteria sets were established [1].

Current classification criteria for pSS were published in 2016 by the American College of Rheumatology (ACR) and the European League Against Rheumatism (EULAR) [2]. They consist of five points (Table I):

- anti-SSA/Ro antibody positivity,

- focal lymphocytic sialadenitis in minor salivary gland biopsy (MSGB) with a focus score $\geq 1$ (over 50 mononuclear cells in one focus in $4 \mathrm{~mm}^{2}$ cross-sectional area),
- an abnormal ocular staining score (or van Bijsterveld score),

- Schirmer's test result $<5 \mathrm{~mm} / 5 \mathrm{~min}$,

- unstimulated whole saliva flow rate below $0.1 \mathrm{ml} / \mathrm{min}$. These criteria replaced the ones from 2012 created by the American College of Rheumatology [3] and the ones from 2002 established by the American-European Consensus Group [4]. Although the diagnostic techniques have developed over time, still there is no evident place for any of the imaging methods in the current classification criteria of pSS.

However, the literature describes studies showing the importance of ultrasound in pSS diagnostic, correlations of changes in ultrasound with the progression/ severity of the inflammatory process in MSGB or the duration of the disease $[5,6]$.

Other imaging tests, such as classical sialography or magnetic resonance sialography, are still applicable to assess the function of the salivary gland, and the introduction of a new non-invasive technique for assessing various organs, which is elastography, may also be useful in assessing salivary gland changes. This article 
Table I. ACR-EULAR Classification Criteria for primary Sjögren's syndrome [2]

\begin{tabular}{|lc|}
\hline Weight & Item \\
\hline 3 & $\begin{array}{r}\text { Labial salivary gland with focal lymphocytic } \\
\text { sialadenitis and focus score } \geq 1\end{array}$ \\
\hline 3 & Anti-SSA (Ro) + \\
\hline 1 & $\begin{array}{c}\text { Ocular staining score } \geq 5 \\
\text { (or van Bijsterveld score } \geq 4) \text { in at least one eye }\end{array}$ \\
\hline 1 & Schirmer $\leq 5 \mathrm{~mm} / 5$ min in at least one eye \\
\hline 1 & Unstimulated whole saliva flow rate $\leq 0.1 \mathrm{ml} / \mathrm{min}$ \\
\hline
\end{tabular}

The classification of SS applies to any individual who meets the inclusion criteria (symptoms of oral or ocular dryness or 1 extra - glandular manifestation of pSS), does not have any conditions considered as exclusion criteria (history of head and neck radiation treatment, active hepatitis C infection (with positive PCR), acquired immunodeficiency syndrome, sarcoidosis, amyloidosis, graft versus host disease, IgG4-related disease), and who has a score $\geq 4$.

discusses available and new methods of imaging the salivary glands useful in the diagnosis of pSS.

\section{Sialography}

Sialography is one of the first imaging methods used for patients with pSS. It was introduced in the 1950s [7]. This method enables the assessment of major salivary gland ducts by the injection of a small amount of contrast medium into the salivary duct, followed by routine $\mathrm{X}$-ray projections. The results are interpreted through the score system of Rubin and Holt (Table II) that consists of five classes of severity and is based both on the morphological abnormalities and the amount of contrast collection $[7,8]$.

Although sialographic examination is an invasive method and it requires the use of an iodized contrast agent, for decades sialography has been the gold standard technique for pSS diagnostics. It is contraindicated in patients with infection, inflammation or allergy to iodine [9].

Additionally, specificity of parotid sialography is not very high as sialographic patterns associated with pSS are also seen in other lympho-epithelial lesions or in recurrent parotitis of childhood [10]. The specificity of sialography ranges from $82.0 \%$ to $100 \%$ and sensitivity ranges from $77.7 \%$ to $95.8 \%[11,12]$. Also, the experience of a radiologist may be crucial to sialography results [12].

As sialography's diagnostic value has its limitations, alternative imaging procedures such as magnetic resonance sialography, dynamic magnetic resonance sialography and computed tomography sialography have been evaluated.

Starting from the 1980s parotid CT sialography was introduced [13]. It was used to indicate surgical landmarks and relationships of the facial nerve to any parotid
Table II. Scoring system of sialography by Rubin and Holt [7]

\begin{tabular}{|c|c|}
\hline Stage & Description \\
\hline Normal image & Without contrast media collection \\
\hline Punctate image & $\begin{array}{l}\text { Refers to contrast media collection } \\
\leq 1 \mathrm{~mm} \text { in diameter }\end{array}$ \\
\hline Globular image & $\begin{array}{l}\text { Contrast media collection between } \\
1 \text { and } 2 \mathrm{~mm} \text { in diameter }\end{array}$ \\
\hline Cavitary image & $\begin{array}{c}\text { Contrast media collection } \geq 2 \mathrm{~mm} \\
\text { in diameter }\end{array}$ \\
\hline Destructive image & $\begin{array}{c}\text { Complete destruction of gland } \\
\text { parenchyma }\end{array}$ \\
\hline
\end{tabular}

masses or to differentiate kinds of tumours rather than to assess the changes in the parotid's structure in pSS.

Later, MR sialography was discovered. This examination is used to assess the ductal system of the parotid salivary gland without using any exogenous contrast agents [14]. It is a non-invasive method which allows one to detect disorders in parotid salivary glands typical for SS. MR sialography allows one to depict the subtle changes in the parotid gland ducts, and specific findings of numerous cystic changes in the salivary glands that represent dilated intraglandular ducts can also be identified on MRI sialograms [15].

Interestingly, the changes seen on MRI sialograms in pSS may precisely reflect progression of the disease $[15,16]$. In the Niemela [17] study MR sialography was compared to ultrasonography and MRI. The author found that it was the most sensitive method among the others despite the fact that the tested sample of patients was relatively small. Both MRI sialography and conventional $\mathrm{MRI}$ are non-invasive methods that provide information of morphologic changes in parotid glands and can be used as diagnostic indicators of pSS. It seems that MR sialography is more sensitive but on the other hand conventional MRI provides information on the pathologic changes of glandular parenchyma [18].

Another technique based on traditional sialography is dynamic magnetic resonance sialography that may noninvasively and quantitatively evaluate the secretion function of parotid glands. In this method images are acquired before and after the administration of citric acid or vitamin C tablets placed on the tongue [19, 20]. The pattern characteristic for pSS is the so-called "apple-tree-like structure" [19].

\section{Scintigraphy}

Major salivary gland scintigraphy is a non-invasive nuclear imaging technique. This method through radioactive tracer infusion of technetium-99 $\left({ }^{99 \mathrm{~m} T \mathrm{C})}\right.$ pertech- 
netate permits evaluation of glandular function by assessing the distribution and speed of elimination of the radio-tracer after secretory stimulation (e.g. lemon juice) [9].

A quantitative score system for scintigraphy was introduced in 1971 by Schall et al. [21] for xerostomia assessment in pSS patients. It consists of grades from 0 to 4 and in 1993 salivary gland scintigraphy was included in pSS criteria in the Preliminary European Classification Criteria for Review Imaging in primary Sjögren's Syndrome [22]. In later criteria from 2002 only some scintigraphy parameters such as delayed uptake, reduced concentration, and/or delayed secretion of the tracer were taken into consideration with no reference to any specific classification score [4].

One reason why the scintigraphy has disappeared from pSS criteria since 2012 may be its moderate sensitivity of up to $89 \%$ and very low specificity of around $50 \%$ [9]. It is related to the abnormal scintigraphy pattern in other major salivary gland diseases characterised by a secretory dysfunction such as chronic recurrent sialodochoadenitis, salivary gland inflammation of a different etiology or simply in involutionary glandular changes in elderly people [23].

Although scintigraphy should not be used to differentiate between pSS and other pathological processes of salivary glands, it should still remain one of the tools of pSS course evaluation. A study conducted by Ramos-Casals et al., in Barcelona [24], on a significant number of 405 pSS patients revealed the association between the degree of involvement shown by parotid scintigraphy and the disease prognosis.

Scintigraphy results were graded from 1 to 4 and patients diagnosed with class 4 involvement (the most severe) had a higher risk of developing parotid enlargement and severe extraglandular manifestations of the disease such as vasculitis or neurological, renal or lung involvement. What is more, patients with class 4 involvement had much higher mortality and a risk of developing B-cell lymphoma.

As the scintigraphy picture and salivary gland function may worsen in some patients with pSS with time, scintigraphy re-examination should be considered in the case of worsening of dryness symptoms. Interestingly, scintigraphy can be used not only to assess the involvement of salivary glands, but also to reveal extra-glandular involvement. To achieve this effect whole-body so-

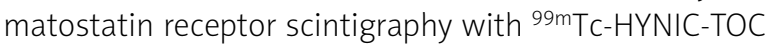
may be performed.

This was the subject of the Anzola [25] research whose results were published in 2019. In this study

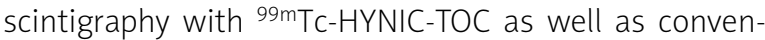
tional sialoscintigraphy with ${ }^{99 \mathrm{~m} T \mathrm{CO} 4}$ was performed on patients with de-novo diagnosis of pSS and control subjects. The examination made it possible to identify active inflammatory processes not only in the salivary glands of pSS patients, but also in the joints [25].

\section{Magnetic resonance imaging}

Magnetic resonance imaging has been used since 1996 to assess salivary glands in SS [26]. This method usually evaluates the appearance of signal intensities on T1- and T2-weighted images. Additionally, fat deposition in the major salivary glands characteristic for this disease can be revealed in MR imaging by using short-inversion-time inversion recovery (STIR) and fat-saturation MR sequences [27].

Magnetic resonance imaging can be advantageous both in early and late stages of the disease. In the initial course of pSS glandular enlargement caused by the oedema may be observed and in the late course there is lobular destruction, associated with deposition of fat and fibrous tissue [9].

On T1 and T2 weighted images the typical feature of salivary glands in pSS is the so-called "salt-and-pepper" appearance that consists of small areas of hypo- and hyper-intensity. In the advanced stages of the disease the pathological condition of salivary glands results in diffuse micro- and/or macrocystic changes, which on T2-weighted images with fat-sat technique or inversion recovery are recognised as hyperintense areas [9].

Although MRI findings of all types of salivary glands may be evaluated, it is considered that the results for the sublingual glands have less diagnostic value for discriminating pSS than for submandibular and parotid glands due to their greater individual and age differences in the size and smaller volume [28]. Furthermore, heterogeneous signal intensity distribution in T1- and fat-suppressed T2-weighted MRI in patients with SS has higher specificity in the submandibular gland than in the parotid gland [28]. The heterogeneity and smaller volume in the submandibular gland observed in MRI may be a good predictor for hyposalivation related to the progression of pSS [29].

Another aspect of pSS that is significant when deciding which imaging method should be chosen not only to diagnose but to assess the stage and activity of the disease is an increased risk of developing lymphoma [1, 9].

Although there is an important overlap between benign and malignant lesions in MRI in each patient with pSS it is vital to pay attention to any dominant, painless mass in salivary glands. In different kind of tumours morphologic features, such as shape and margins, exhibit considerable similarity. 
Generally, almost all masses are hypo-intense compared to salivary glands on non-enhanced T1 weighted images, and on contrast-enhanced images almost all salivary gland neoplasms enhance. On T2-weighted images usually hyper-intense masses seem to be benign, while intermediate and hypointense lesions seem to be malignant. As most of these features are non-specific in many cases cytological or histological assessment is obligatory [9].

Fortunately, the location of the salivary glands makes tissue sampling relatively simple to perform and radiologic diagnosis is not so highly demanded [30] However, MRI still can be used in local staging as most lymphomas initially involve the neck organs. MR imaging focused on the cervical regions can be used in the follow-up [31].

\section{Ultrasonography}

Salivary gland ultrasonography (SGUS) has been used for the diagnosis and follow-up of pSS for more than 25 years [32]. It has an established role in detecting typical structural salivary gland abnormalities characteristic for this disease. Currently, B-mode SGUS is one of the most often used imaging methods to assess structural changes of the salivary glands. It is a noninvasive, inexpensive, accessible, repeatable test without exposure to ionizing radiation.

Since 1992 there have been a few SGUS scores developed [33] and they exist as many different variants. The most often used scoring systems are the one developed by De Vita [32] and the simplified scoring system proposed by Theander a few years ago [34]. The first one assesses 4 salivary glands (2 submandibular and 2 parotid) with scores from 0 to 4 and the final score is the sum of the scores of the 4 glands (0-16). The simplified version is based on the homogeneity of glands. The glands are scored from 0 to 4 and the highest score in any of the 4 salivary glands is set as the final score [34].

As there still exist various scoring systems in 2012 the ACR/EULAR aimed to standardize SGUS in order to maximize its usefulness in pSS. There has been an international consensus reached to define echostructural abnormalities characteristic for pSS and to set up a preliminary atlas of changes in salivary glands [35].

Furthermore, in 2016 the European Community funded a project called "HarmonicSS" [36] dedicated to patients with pSS [33]. This project aimed to develop different and novel imaging approaches in pSS and there was also an attempt to use artificial intelligence for salivary gland damage assessment by SGUS in pSS.

So far many studies have demonstrated the accuracy and reliability of using ultrasonography in diagnosing and differentiating pSS from non-pSS sicca syndrome.
Although ultrasound imaging has never been included in pSS criteria there have been attempts to make this method widespread and consider its accuracy in diagnosing process.

One of the biggest cohort studies assessing whether addition of SGUS to pSS criteria or replacement of current criteria items by SGUS would influence its accuracy was conducted between 2014 and 2017 in the Netherlands [37]. The primary aim of the cited study was to evaluate whether presence of hypoechogenic areas as a criterion item influences the performance of pSS criteria. There were 243 patients included (147 pSS patients and 96 non-pSS). All of them underwent salivary gland biopsy (labial, parotid or both) as well as salivary gland ultrasonography. Each SGUS result was scored from 0 to 3 in the submandibular and parotid gland on the right side depending on the presence of hypoechogenic areas: 0 for no hypoechogenic areas, 1 for a few scattered areas, 2 for several areas, and 3 for numerous hypoechogenic areas. The statistical analysis revealed that a good distinction between pSS and non-pSS patients was the average SGUS score for hypoechogenic areas $\geq 1.5$. This optimal cut-off value of SGUS $\geq 1.5$ corresponds to sensitivity of $72.1 \%$ and specificity of $93.8 \%$.

What is more, if positive SGUS was added to the pSS criteria with a weight of 1 point the optimal cut-off point of the modified ACR/EULAR score to discriminate between pSS and non-pSS would be $\geq 4$ (as it is in the current criteria). The authors suggested that SGUS can possibly replace the ocular staining score, Schirmer's test or unstimulated whole saliva flow in the classification criteria but still the salivary gland biopsy or the measurement of anti-SSA antibodies remains the most important when making the diagnosis. However, it seems that SGUS should be analyzed together with the other components of the criteria in order to increase the accuracy of diagnosis.

\section{Elastography}

Elastography is another non-invasive imaging technique that is used to investigate the elasticity of soft tissues. It was first described in the 1990s, and since then it has been developed to enable qualitative and quantitative assessment of tissue stiffness [38, 39]. Elastography monitors tissue mechanical properties by measuring the response of examined tissue to acoustic energy. This method is based on the changed elasticity of soft tissues resulting from some specific physiological or pathological processes and is becoming widely used to differentiate affected from normal tissue [38]. There are two main types of elastography: strain imaging and shear wave imaging $[40,41]$. 
In strain imaging tissue stiffness is measured by applying external tissue pressure. The stiffer the examined tissue is, the less it deforms. There are a few subtypes of strain imaging: strain elastography (i.e. real time tissue elastography, Elastoscan) with manual compression and ARFI imaging (i.e. Virtual Touch Imaging) with acoustic radiation force impulse excitation [40]. Shear wave imaging is a quantitative method which exploits the fact that shear waves propagate faster in hard than soft tissue. What is important, shear waves propagate in a direction orthogonal to the direction of the tissue displacement, not as ultrasonic waves that propagate in the same direction as the tissue displacement. Among shear wave imaging it is possible to distinguish point shear wave speed measurement (i.e. Virtual Touch Quantification - VTQ), shear wave speed imaging (both of them with acoustic radiation force impulse excitation) and transient elastography (i.e. Fibroscan) with controlled external vibration as an excitation method [40].

The most common use of elastography is to evaluate liver fibrosis and renal fibrosis, characterise breast lesions, and assess thyroid nodules and lymph nodes $[38,42]$. Less frequent applications include detection of ulnar neuropathy [43]. There are also studies showing the possible use of elastography in SS.

One of the first articles on this topic was published in 2014 [44]. It presented the results of a study conducted in Austria based on real-time tissue elastography (RTTE) (a version of strain elastography) compared with the results of B-mode ultrasonography. There were 45 pSS patients, 24 sicca patients without confirmation of pSS and 11 healthy subjects. The study showed that RTTE may be a valuable tool in pSS syndrome diagnosis and it can be used for the assessment of functional impairment of salivary glands.

Almost at the same time also shear wave imaging was implemented as an imaging technique for pSS [42]. One of the first studies on that issue tried to compare patients with sialolithiasis (33), Stensen's duct stenosis (15), chronic inflammation (10), pSS (20) and healthy subjects in terms of parotid glands elasticity [42]. Extraordinary high stiffness measured in kilopascals $(\mathrm{kPa})$ was discovered in pSS compared to other groups and that encouraged investigators to perform further studies. Additionally, some data indicate that elastography may also be a possible diagnostic tool for identifying early pSS changes in the salivary glands [45].

Other results were presented in a study published in 2016 which aimed to compare real-time tissue elastography, Virtual Touch Imaging (VTI) (both of them are versions of strain imaging) and VTQ (shear-wave imaging) in patients with pSS and non-pSS sicca syndrome [46] These results suggest that the application of strain elas- tography techniques such as RTTE and VTI seems to be non-significant with respect to the evaluation of salivary glands in PSS, whereas VTQ may be useful in identifying early stages of pSS.

What is more, in another study in patients with pSS it was possible to correlate the results of shear wave velocity (SWV) elastography with the B-mode ultrasound score and systemic and glandular activity of the disease, and increased SWV might represent chronic glandular inflammation [47].

Shear wave imaging is a reliable, non-invasive, cost-effective modality for the assessment of generalised parotid gland diseases such as pSS but still further studies on this issue should be conducted.

\section{Conclusions}

Although not included in current classification criteria for pSS, imaging techniques provide additional information about the involvement of the major salivary glands.

Nowadays it seems that SGUS is more and more often used and in the future it may become a valid instrument to ameliorate pSS diagnosis and evaluation. Salivary gland ultrasonography with its high sensitivity and specificity, accessibility, non-invasive characteristics, repeatability and low cost is commonly used in clinical practice but it still lacks widely approved international standardization.

Elastography, especially shear wave imaging, also seems to be an interesting and promising technique that may distinguish chronic glandular inflammation from a normal glandular tissue. It is an objective method but so far no studies on big cohorts of patients have been conducted.

Magnetic resonance imaging may be used both in early and advanced stages of the disease. It is especially useful in detection of lymphoma, which is one of the most serious complications of pSS. However, MRI remains an expensive method and it is contradicted in some patients due to the use of a magnetic field.

Functional assessment of salivary glands provided by scintigraphy although not useful when differentiating between pSS and other pathological processes may be beneficial to assess intensity of xerostomia and severity of salivary gland dysfunction reported by patients.

Sialography due to its invasiveness and the need of contrast medium application is not very often used in pSS patients but it still remains an important examination for other pathological conditions such as sialolithiasis. It seems that traditional sialography in future may be replaced by more modern techniques based on it such as magnetic resonance sialography or based on other mixed techniques. 
All of the methods mentioned above provide different information about the condition of salivary glands in pSS. Unfortunately, it is impossible to compare their use in clinical practice and it is difficult to evaluate different studies conducted on pSS patients as they were based on various pSS classification criteria and statistically valid groups of patients were not always examined.

The existing data and ongoing research deal with the challenge of establishing the best imaging method to evaluate damage in major salivary glands, which are the most commonly affected organs in pSS.

The authors declare no conflict of interest.

\section{References}

1. Vivino FB. Sjögren's syndrome: Clinical aspects. Clin Immunol 2017; 182: 48-54.

2. Shiboski CH, Shiboski SC, Seror R, et al. 2016 American College of Rheumatology/European League Against Rheumatism Classification Criteria for Primary Sjögren's Syndrome: A Consensus and Data-Driven Methodology Involving Three International Patient Cohorts. Arthritis Rheumatol 2017; 69: 35-45.

3. Shiboski SC, Shiboski CH, Criswell LA, et al. American College of Rheumatology classification Criteria for Sjögren's Syndrome: a Data-Driven, Expert Consensus Approach in the SICCA Cohort. Arthritis Care Res (Hoboken) 2012; 64: 475-87.

4. Vitali C, Bombardieri S, Jonsson R, et al. Classification criteria for Sjögren's syndrome: a revised version of the European criteria proposed by the American-European Consensus Group. Ann Rheum Dis 2002; 61: 554-558.

5. Baldini C, Luciano N, Tarantini G, et al. Salivary gland ultrasonography: a highly specific tool for the early diagnosis of primary Sjögren's syndrome. Arthritis Res Ther 2015; 17: 146.

6. Cornec D, Jousse-Joulin S, Pers JO, et al. Contribution of salivary gland ultrasonography to the diagnosis of Sjögren's syndrome: toward new diagnostic criteria? Arthritis Rheum 2013; 65: 216-225.

7. Rubin P, Holt JF. Secretory sialography in diseases of the major salivary glands. Am J Roentgenol Radium Ther Nucl Med 1957; 77: 575-598.

8. de Sousa Gomes P, Juodzbalys G, Fernandes MH, Guobis Z. Diagnostic Approaches to Sjögren's syndrome: a Literature Review and Own Clinical Experience. J Oral Maxillofac Res 2012; 3: e3.

9. Baldini C, Zabotti A, Filipovic N, et al. Imaging in primary Sjögren's syndrome: the "obsolete and the new". Clin Exp Rheumatol 2018; 36 (Suppl 112): 215-221.

10. Cutolo M, Damjanov N, Ruaro B, et al. Imaging of connective tissue diseases: Beyond visceral organ imaging? Best Pract Res Clin Rheumatol 2016; 30: 670-687.

11. Song GG, Lee YH. Diagnostic accuracies of sialography and salivary ultrasonography in Sjögren's syndrome patients: a metaanalysis. Clin Exp Rheumatol 2014; 32: 516-522.

12. Kalk WW, Vissink A, Spijkervet FK, et al. Parotid sialography for diagnosing Sjogren syndrome. Oral Surg Oral Med Oral Pathol Oral Radiol Endod 2002; 94: 131-137.
13. Stone DN, Mancuso AA, Rice D, Hanafee WN. Parotid CT sialography. Radiology 1981; 138: 393-397.

14. Ogura I, Sasaki Y, Oda T, et al. Magnetic Resonance Sialography and Salivary Gland Scintigraphy of Parotid Glands in Sjögren's Syndrome. Chin J Dent Res 2018; 21: 63-68.

15. Tonami H, Oqawa Y, Matoba M, Kuqinuki Y, et al. MR sialography in patients with Sjögren syndrome. AJNR Am J Neuroradiol 1998; 19: 1199-1203.

16. Reqier M, Ries T, Arndt C, et al. Sjögren's syndrome of the parotid gland: value of diffusion-weighted echo-planar MRI for diagnosis at an early stage based on MR sialography grading in comparison with healthy volunteers. Rofo 2009; 181: 242-248.

17. Niemelä RK, Takalo R, Pääkkö E, Suramo I, et al. Ultrasonography of salivary glands in primary Sjögren's syndrome. A comparison with magnetic resonance imaging and magnetic resonance sialography of parotid glands. Rheumatology (Oxford) 2004; 43: 875-879.

18. Niemelä RK, Takalo R, Pääkkö E, et al. .Magnetic resonance imaging and magnetic resonance sialography of parotid glands in primary Sjögren's syndrome. Arthritis Rheum 2001; 45: 512-518.

19. Morimoto $\mathrm{Y}, \mathrm{Habu}$ M, Tomoyose T, et al. Dynamic magnetic resonance sialography as a new diagnostic technique for patients with Sjögren's syndrome. Oral Dis 2006; 12: 408-414.

20. Liu S, Chen W, Wang M, et al. Quantitative Analysis of Parotid Gland Secretion Function in Sjögren's Syndrome Patients with Dynamic Magnetic Resonance Sialography. Korean J Radiol 2019; 20: 498-504.

21. Schall GL, Anderson LG, Wolf RO, et al. Xerostomia in Sjögren's syndrome. Evaluation by sequential salivary scintigraphy. JAMA 1971; 216: 2109-2116.

22. Vitali C, Bombardieri S, Moutsopoulos HM, et al. Preliminary criteria for the classification of Sjögren's syndrome. Results of a prospective concerted action supported by the European Community. Arthritis Rheum 1993; 36: 340-347.

23. Salaffi F, Carotti M, lagnocco A, et al. Ultrasonography of salivary glands in primary Sjögren's syndrome: a comparison with contrast sialography and scintigraphy. Rheumatology (Oxford) 2008; 47: 1244-1249.

24. Ramos-Casals M, Brito-Zerón P, Perez-DE-Lis M, et al. Clinical and prognostic significance of parotid scintigraphy in 405 patients with primary Sjögren's syndrome. J Rheumatol 2010; 37: 585-590.

25. Anzola LK, Rivera JN, Dierckx RA, et al. Value of Somatostatin Receptor Scintigraphy with 99mTc-HYNIC-TOC in Patients with Primary Sjögren Syndrome. J Clin Med 2019; 8; 763.

26. Izumi M, Equchi K, Ohki M, et al. MR imaging of the parotid gland in Sjögren's syndrome: a proposal for new diagnostic criteria. AJR Am J Roentgenol 1996; 166: 1483-1487.

27. Izumi M, Equchi K, Nakamura $\mathrm{H}$, et al. Premature fat deposition in the salivary glands associated with Sjögren syndrome: MR and CT evidence. AJNR Am J Neuroradiol 1997; 18: 951-958.

28. Kojima I, Sakamoto M, likubo M, et al. Diagnostic performance of MR imaging of three major salivary glands for Sjögren's syndrome. Oral Dis 2017; 23: 84-90.

29. Kojima I, Sakamoto M, likubo M, et al. Relationship of MR imaging of submandibular glands to hyposalivation in Sjögren's syndrome. Oral Dis 2019; 25: 117-125. 
30. Kessler AT, Bhatt AA. Review of the Major and Minor Salivary Glands, Part 2: Neoplasms and Tumor-like Lesions. J Clin Imaging Sci 2018; 8: 48.

31. Tonami H, Matoba M, Kuginuki Y, et al. Clinical and imaging findings of lymphoma in patients with Sjögren syndrome. J Comput Assist Tomogr 2003; 27: 517-524.

32. De Vita, Lorenzon G, Rossi G, et al. Salivary gland echography in primary and secondary Sjögren's syndrome. Clin Exp Rheumatol 1992; 10: 351-356.

33. Devauchelle-Pensec V, Zabotti A, Carvajal-Alegria G, et al. Salivary gland ultrasonography in primary Sjögren's syndrome: opportunities and challenges. Rheumatology (Oxford) 2019; pii: kez079.

34. Theander E, Mandl T. Primary Sjögren's syndrome: diagnostic and prognostic value of salivary gland ultrasonography using a simplified scoring system. Arthritis Care Res (Hoboken) 2014; 66: 1102-1107.

35. Jousse-Joulin S, Nowak E, Cornec D, et al. Salivary gland ultrasound abnormalities in primary Sjögren's syndrome: consensual US-SG core items definition and reliability. RMD Open 2017; 3: e000364.

36. HarmonicSS. https://www.harmonicss.eu/

37. van Nimwegen JF, Mossel E, Delli K, et al. Incorporation of salivary gland ultrasonography into the ACR-EULAR criteria for primary Sjögren's syndrome. Arthritis Care Res (Hoboken) 2019 [Epub ahead of print].

38. Sigrist RMS, Liau J, Kaffas AE, et al. Ultrasound Elastography: Review of Techniques and Clinical Applications. Theranostics 2017; 7: 1303-1329.

39. Gennisson JL, deffieux T, Fink M, et al. Ultrasound elastography: principles and techniques. Diagn Interv Imaging 2013; 94: 487-495.
40. Shiina T, Nightingale KR, Palmeri $M L$, et al. WFUMB guidelines and recommendations for clinical use of ultrasound elastography: Part 1: basic principles and terminology. Ultrasound Med Biol 2015; 41: 1126-1147.

41. Ozturk A, Grajo JR, Dhyani M, et al. Principles of ultrasound elastography. Abdom Radiol (NY) 2018; 43: 773-785.

42. Wierzbicka $M$, Kałużny J, Ruchała $M$, et al. Sonoelastography - a useful adjunct for parotid gland ultrasound assessment in patients suffering from chronic inflammation. Med Sci Monit 2014; 20: 2311-2317.

43. Paluch $七$, Noszczyk B, Nitek $\dot{Z}$, et al. Shear-wave elastography: a new potential method to diagnose ulnar neuropathy at the elbow. Eur Radiol 2018; 28: 4932-4939.

44. Dejaco C, de Zordo T, Heber D, et al. Real-time sonoelastography of salivary glands for diagnosis and functional assessment of primary Sjögren's syndrome. Ultrasound Med Biol 2014; 40: 2759-2767.

45. Chen S, Wang Y, Chen S, et al. Virtual Touch Quantification of the Salivary Glands for Diagnosis of Primary Sjögren Syndrome. J Ultrasound Med 2016; 35: 2607-2613.

46. Hofauer B, Mansoyr N, Heiser C, et al. Sonoelastographic Modalities in the Evaluation of Salivary Gland Characteristics in Sjögren's Syndrome. Ultrasound Med Biol 2016; 42: 21302139.

47. Kimura-Hayama E, Criales-Vera S, Azpeitia-Espinosa L, et al. Elastographic ultrasound: an additional image tool in Sjögren's syndrome. Int J Rheum Dis 2018; 21: 1293-1300. 